\title{
Potential Application of Dandelion (Taraxacum Mongolicum Hand.-Mazz.) on Broiler Chickens: Growth Performance, Expression of Tight Junction Protein and Mucin Genes, and Microbiota Composition in lleum
}

\section{Jinju Mao}

College of Animal Science,Inner Mongolia Agricultural University, Hohhot 010018, China

Yuan Wang ( $\sim$ wangyuan.926@163.com )

College of Animal Science,Inner Mongolia Agricultural University, Hohhot 010018, China

Wenwen Wang

College of Animal Science,Inner Mongolia Agricultural University, Hohhot 010018, China

Ting Duan

College of Animal Science,Inner Mongolia Agricultural University, Hohhot 010018, China

$\mathrm{Na}$ Yin

College of Animal Science,Inner Mongolia Agricultural University, Hohhot 010018, China

Tao Guo

College of Animal Science,Inner Mongolia Agricultural University, Hohhot 010018, China

Hui Guo

College of Animal Science,Inner Mongolia Agricultural University, Hohhot 010018, China

$\mathrm{Na}$ Liu

College of Animal Science,Inner Mongolia Agricultural University, Hohhot 010018, China

Xiaoping An

College of Animal Science,Inner Mongolia Agricultural University, Hohhot 010018, China

Jingwei Qi

College of Animal Science,Inner Mongolia Agricultural University, Hohhot 010018, China

\section{Research Article}

Keywords: dandelion, growth performance, barrier function, microbiota composition, broiler

Posted Date: February 28th, 2022

DOI: https://doi.org/10.21203/rs.3.rs-1299373/v1

License: (c) (i) This work is licensed under a Creative Commons Attribution 4.0 International License. Read Full License 


\section{Abstract \\ Background}

Chinese herbs is becoming an exploitable alternative to the use of widely prohibited antibiotics in the poultry industry. We investigated the effects of dandelion on the growth performance and intestinal barrier function of broiler chickens. One-hundred and sixty 1-day-old Arbor Acres (AA) male broilers were randomly divided into four groups, with five replicates of eight birds each. The birds were fed a basal diet supplemented without (control group, [CON]) or with 500 (low dose [LD]) or 1000 (high dose [HD]) $\mathrm{mg} / \mathrm{kg}$ dandelion or with $50 \mathrm{mg} / \mathrm{kg}$ chlortetracycline (CTC) for 42 days, including the starter phase (d 1 to 21 ) and the grower phase (d 22 to 42 ).

\section{Results}

During the starter phase, the average daily feed intake (ADFI) and feed/gain ratio $(F / G)$ of LD was lower than CON, HD and CTC $(P<0.05)$. In the overall phase, the F/G of LD and HD both decreased $(P<0.05)$. At 21 days old, the gene expression of claudin, occludin-1, and mucin-1 of the LD and HD birds were significantly higher than that of CON and CTC birds $(P<0.05)$; the gene expression of claudin and occludin- 1 was significantly higher in HD birds than in LD birds; and the gene expression of zonula occludens-1 (ZO-1) in HD birds was higher than that of CON and LD birds, but lower than that of CTC birds (all $P<0.05$ ). At 42 days old, the gene expression of claudin, occludin-1, and mucin-1 of the LD birds was significantly higher than that of the other groups, whereas the gene expression of zo-1 was higher in the HD birds than in CON and LD birds (all $P<0.05$ ). In the analysis of intestinal microbiota, decreased a-diversity was observed in 21-days-old HD birds, while LD and HD birds showed a significantly decreased relative abundance of Bacteroidata and Bacteroides at the level of phyla and genera $(P<$ 0.05).

\section{Conclusion}

Overall, dietary dandelion supplementation can enhance growth performance by improving the intestinal barrier function of broilers. Therefore, dandelion represents a promising feed additive for broilers, although the optimal supplementation dose requires further study.

\section{Background}

In modern poultry farming, broilers were raised under highly intensive production systems, which cause various diseases due to high-density [1,2]. To prevent diseases and promote growth, antibiotics have been widely used in broiler production $[3,4]$. However, the abuse of antibiotics has resulted in serious consequences, such as microbial dysbiosis, organism immunity dysfunction, antimicrobial resistance, and antibiotic residues, all of which represent serious threats to the health of both animals and humans $[5,6,7]$. Therefore, these issues led to the ban of antibiotic growth promoters as feed additives in husbandry production. According to the Ministry of Agriculture and Rural Affairs Announcement No. 194, from July 1, 2020, China completely banned the use of antibiotic growth promoter additives for livestock production.

The complete intestinal barrier is a complex structure made up of physical, chemical, immunological, and microbiological barriers, all of which play pivotal roles preventing pathogens and toxins from entering the animal 
and causing disease $[8,9]$. Hence, the intactness of intestinal barrier function is crucial for the wellness and productivity of broilers. Therefore, the identification of feed additives that are suitable for improving the intestinal barrier function and alternating antibiotics for poultry production is becoming increasingly urgent.

Chinese herbs $(\mathrm{CH})$ have gained widespread attention as alternatives to antibiotic growth promoter additives for their potential to modulate nutritional metabolism, immune response, and intestinal health [10]. $\mathrm{CH}$ also have advantages of extensive sources and limited side effects. Previous studies have reported that $\mathrm{CH}$ can enhance growth performance by improving intestinal mucosal barrier function, such as by increasing the mRNA expression of tight junction proteins, developing intestinal immunity, and improving the ecology of gut microbiota and so on $[11,12,13,14]$.

Dandelion (Taraxacum mongolicum Hand.-Mazz.) is a kind of $\mathrm{CH}$ with a long history in China [15]. Dandelion contains active substances, such as polysaccharides, polyphenols and terpenoids, as well as numerous nutrients, including minerals, proteins, vitamins and trace elements [16]. It has been proven that dandelion displays antiinflammatory and antibacterial activities in vitro $[17,18]$. A previous study demonstrated that dandelion root extracts can improve the growth performance of pigs reflected by an increased average daily gain [19]. Research on golden pompano Trachinotus ovatus has shown that diet supplementation with dandelion extracts improved growth performance by improving the richness and diversity of gut microbiota. Furthermore, dietary dandelion extract has been shown to enhance intestinal barrier function and immunity by regulating the mRNA levels of tight junction proteins and immune-related genes [20,21]. However, no study has been conducted to examine the effects of dandelion on growth performance and intestinal barrier functions of broilers. Therefore, we aimed to test the hypothesis that dandelion could enhance the growth performance of broilers by improving intestinal barrier function.

\section{Results}

\section{Growth performance}

The growth performance of broilers is presented in Figs. 1-4. Figure 1 shows that there was no significant difference in the initial weight of birds between groups $(P>0.05)$, and there was no significant difference in weight between groups at 21 and 42 days old $(P>0.05)$. Figure 2 shows that compared to $C O N$, there was no significant change in ADG with LD and HD at the starter, grower, and whole phase $(P>0.05)$. Compared to $C T C$, the ADG with LD birds increased in the grower and whole phases $(P<0.05)$. Figure 3 shows that the ADFI of the LD birds was lower than that of the CON and HD birds in the starter phase $(P<0.05)$. Moreover, the ADFI of the CON birds was higher than that of the CTC birds in the grower and whole phases $(P<0.05)$. The ADFI of the LD birds was lower than that of the CTC birds in the starter phase $(\mathrm{P}<0.05)$, but significantly higher than the CTC birds in the grower phase $(P<0.05)$. The results of $F / G$ are revealed in Fig. 4: compared to $C O N$, the LD birds showed a significantly decreased $F / G$ in the starter and whole phases $(P<0.05)$, while the HD birds showed a significantly decreased $F / G$ in the whole phase $(P<0.05)$. Compared to the CTC birds, the F/G of the LD birds was decreased in the starter phase $(P<0.05)$.

\section{Gene expression of tight junction proteins in ileum}

The effects of dandelion on the gene expression of tight junction proteins in ileum of broilers are showed in Table 1. At 21 days old, the gene expression of claudin and occludin-1 was significantly increased in LD and HD birds compared to CON birds, and that was increased in HD birds compared to LD birds $(P<0.05)$. Moreover, the zonula 
occludens-1 (ZO-1) gene expression of HD birds was higher than that in CON and LD birds $(P<0.05)$. Compared to the CTC group, the expression of claudin and occludin- 1 genes in the LD and HD groups was increased $(P<0.05)$, while that of ZO-1 in was decreased in the CON and LD groups $(P<0.05)$. At 42 days old, the gene expression of claudin and occludin-1 in the LD group was higher than that in the CON and HD groups $(P<0.05)$, while the gene expression of ZO-1 was higher in the HD group than in the CON and LD groups $(P<0.05)$. As revealed by contrast analysis, the gene expression of claudin in LD and occludin-1 in CON and LD birds was higher than that in CTC birds $(P<0.05)$, and the gene expression of ZO- 1 in CON and LD birds was lower than that in CTC birds $(P<0.05)$.

Table 1

Effects of dandelion on gene expression of intestinal tight junction proteins of broilers.

\begin{tabular}{|c|c|c|c|c|c|c|c|c|c|}
\hline \multirow[t]{2}{*}{ Items } & \multicolumn{4}{|c|}{ Treatment group } & \multirow[t]{2}{*}{ SEM } & \multicolumn{4}{|l|}{ P-value } \\
\hline & CON & LD & HD & CTC & & ANONA & $\begin{array}{l}\text { CON vs } \\
\text { CTC }\end{array}$ & $\begin{array}{l}\text { LD vs } \\
\text { CTC }\end{array}$ & $\begin{array}{l}\text { HD vs } \\
\text { CTC }\end{array}$ \\
\hline \multicolumn{10}{|l|}{$\begin{array}{l}21 \text { days } \\
\text { old }\end{array}$} \\
\hline Claudin & $1.012^{c}$ & $2.277^{b}$ & $3.854^{a}$ & 0.638 & 0.3182 & $<0.001$ & 0.211 & $<0.001$ & $<0.001$ \\
\hline Occludin-1 & $1.010^{c}$ & $2.187^{b}$ & $3.626^{a}$ & 0.700 & 0.2954 & $<0.001$ & 0.324 & $<0.001$ & $<0.001$ \\
\hline ZO-1 & $0.931^{b}$ & $0.690^{b}$ & $1.484^{\mathrm{a}}$ & 2.000 & 0.1491 & $<0.001$ & $<0.001$ & $<0.001$ & 0.038 \\
\hline \multicolumn{10}{|l|}{$\begin{array}{l}42 \text { days } \\
\text { old }\end{array}$} \\
\hline Claudin & $1.008^{b}$ & $2.313^{a}$ & $1.359^{b}$ & 0.932 & 0.1513 & $<0.001$ & 0.706 & $<0.001$ & 0.059 \\
\hline Occludin-1 & $1.003^{b}$ & $1.382^{a}$ & $0.764^{b}$ & 0.622 & 0.0846 & 0.009 & 0.022 & $<0.001$ & 0.376 \\
\hline ZO-1 & $1.063^{b}$ & $1.220^{\mathrm{b}}$ & $2.719^{a}$ & 2.638 & 0.1903 & $<0.001$ & $<0.001$ & $<0.001$ & 0.666 \\
\hline
\end{tabular}

a,b,c Means $(n=5)$ within a row with different letters differed significantly $(P<0.05)$; SEM = standard error of the mean.

$\mathrm{CON}=$ the control treatment, $\mathrm{LD}=$ the low dose of dandelion treatment, $\mathrm{HD}=$ the high dose of dandelion treatment, $\mathrm{CTC}=$ the chlortetracycline treatment. ANONA = the treatment effect of CON, LD and HD; CON vs CTC/ LD vs CTC/ HD vs CTC = contrasts comparison of the chlortetracycline group with groups of different additive doses of dandelion.

\section{Gene expression of mucin-1 (MUC1) in ileum}

The expression of MUC1 in broilers fed a diet supplemented with dandelion is shown in Fig. 5. At 21 days old, compared to $\mathrm{CON}$, the gene expression of MUC1 was increased when dandelion was added to the diet $(P<0.05)$, more so in HD birds than in LD birds. Contrast analysis revealed that the gene expression of MUC1 in LD and HD birds was higher than that in CTC birds $(P<0.05)$. At 42 days old, the gene expression of MUC1 was higher in the LD group than that in the CON and HD groups $(\mathrm{P}<0.05)$. Compared to the CTC group, MUC1 gene expression was increased in the CON and LD groups $(P<0.05)$.

\section{Ileal tissue immunity}

Table 2 shows the effects of dandelion on the ileal tissue immunity of broilers. At 21 days old, there was no significant difference between the CON, LD and HD groups, and the content of TNF-a in the LD and HD groups was 
lower than that in the CTC group $(P<0.05)$. At 42 days old, compared to the CON group, the content of SIgA in HD birds was increased by $15.95 \%(P>0.05)$, whereas compared to the CTC group, the content of SIgA in the LD birds was decreased $(P<0.05)$.

Table 2

Effects of dandelion on the intestinal tissue immunity of broilers ( $\mathrm{ng} / \mathrm{g}$ protein)

\begin{tabular}{|c|c|c|c|c|c|c|c|c|c|}
\hline \multirow[t]{2}{*}{ Items } & \multicolumn{4}{|c|}{ Treatment group } & \multirow[t]{2}{*}{ SEM } & \multicolumn{4}{|l|}{$P$-value } \\
\hline & CON & LD & HD & СтC & & ANONA & $\begin{array}{l}\text { CONvs } \\
\text { CTC }\end{array}$ & $\begin{array}{l}\text { LD vs } \\
\text { CTC }\end{array}$ & $\begin{array}{l}\text { HD vs } \\
\text { CTC }\end{array}$ \\
\hline \multicolumn{10}{|c|}{$\begin{array}{l}21 \text { days } \\
\text { old }\end{array}$} \\
\hline IL-1 & 40.02 & 36.98 & 39.74 & 42.60 & 1.465 & 0.737 & 0.571 & 0.227 & 0.531 \\
\hline IL-2 & 96.85 & 85.01 & 77.17 & 82.13 & 4.925 & 0.440 & 0.307 & 0.848 & 0.727 \\
\hline IL-10 & 31.25 & 43.83 & 41.18 & 43.47 & 2.720 & 0.238 & 0.123 & 0.962 & 0.762 \\
\hline SIgA & 379.43 & 405.13 & 424.38 & 428.07 & 18.889 & 0.715 & 0.383 & 0.695 & 0.950 \\
\hline TNF-a & 44.86 & 35.81 & 34.75 & 49.25 & 2.219 & 0.223 & 0.385 & 0.022 & 0.014 \\
\hline \multicolumn{10}{|c|}{$\begin{array}{l}42 \text { days } \\
\text { old }\end{array}$} \\
\hline IL-1 & 37.97 & 35.05 & 45.21 & 39.09 & 2.364 & 0.257 & 0.872 & 0.560 & 0.381 \\
\hline IL-2 & 66.75 & 73.81 & 80.64 & 66.35 & 4.254 & 0.518 & 0.976 & 0.548 & 0.258 \\
\hline IL-10 & 37.71 & 36.56 & 37.81 & 35.81 & 2.088 & 0.980 & 0.779 & 0.906 & 0.754 \\
\hline SIgA & 381.33 & 371.96 & 442.15 & 476.59 & 18.078 & 0.362 & 0.064 & 0.034 & 0.456 \\
\hline TNF-a & 39.50 & 37.85 & 38.43 & 38.07 & 1.905 & 0.962 & 0.810 & 0.970 & 0.951 \\
\hline \multicolumn{10}{|c|}{$\begin{array}{l}a, b \text { Means }(n=5) \text { within a row with different letters differed significantly }(P<0.05) \text {; SEM = standard error of the } \\
\text { mean. }\end{array}$} \\
\hline \multicolumn{10}{|c|}{$\begin{array}{l}C O N=\text { the control treatment, } L D=\text { the low dose of dandelion treatment, } H D=\text { the high dose of dandelion } \\
\text { treatment, } C T C=\text { the chlortetracycline treatment. } A N O N A=\text { the treatment effect of } C O N, L D \text { and } H D ; C O N \text { v } C T C / \\
L D \text { VS } C T C / H D \text { S } C T C=\text { contrasts comparison of the chlortetracycline group with groups of different additive } \\
\text { doses of dandelion. }\end{array}$} \\
\hline
\end{tabular}

\section{lleal microbiota}

The alpha diversity of ileal microbiota was examined to analyze the complexity of species diversity through the Observed_species, Shannon, Simpson, Ace, and Chao1 indices (Table 3). At 21 days old, compared to the CON group, the Observed_species, Simpson, Ace and Chao1 indices were decreased in the HD group $(P<0.05)$, while the Shannon indices were decreased in the LD and HD groups $(P<0.05)$. Compared to the CTC group, the indices of alpha diversity in CON were higher $(P<0.05)$, and there was no significant change in $L D$ and HD groups $(P>0.05)$. At 42 days old, there was no significant difference between the CON, LD and HD groups $(P>0.05)$, compared to the CTC group, the Observed_species in LD group and the Shannon and Simpson indices in CON group were decreased $(P<0.05)$. The principal coordinates analysis $(P C o A)$ reflected the beta diversity by overall microbial profiles of all 
the groups. PCoA of ileum microbiota based on Bray-Curtis distance revealed that there were no significant differences for the bacrerial communities among the 4 groups at 21 and 42 days old (Fig. 6-7).

Table 3

Effects of dandelion on the a-diversity of the ileal content of bacterial communities.

\begin{tabular}{|c|c|c|c|c|c|c|c|c|c|}
\hline \multirow[t]{2}{*}{ Items } & \multicolumn{4}{|c|}{ Treatment group } & \multirow[t]{2}{*}{ SEM } & \multicolumn{4}{|l|}{ P-value } \\
\hline & CON & LD & HD & CTC & & ANONA & $\begin{array}{l}\text { CON } \\
\text { vs } \\
\text { CTC }\end{array}$ & $\begin{array}{l}\text { LD vs } \\
\text { CTC }\end{array}$ & $\begin{array}{l}\text { HD } \\
\text { vs } \\
\text { CTC }\end{array}$ \\
\hline \multicolumn{10}{|l|}{21 days old } \\
\hline Observed_species & $277^{a}$ & $178^{a b}$ & $136^{b}$ & 135 & 20.226 & 0.043 & 0.008 & 0.371 & 0.977 \\
\hline Shannon & $4.05^{a}$ & $2.86^{b}$ & $2.32^{b}$ & 2.80 & 0.204 & 0.010 & 0.012 & 0.893 & 0.299 \\
\hline Simpson & $0.87^{a}$ & $0.72^{\mathrm{ab}}$ & $0.62^{b}$ & 0.74 & 0.031 & 0.016 & 0.069 & 0.832 & 0.101 \\
\hline Ace & $280.76^{a}$ & $184.36^{a b}$ & $142.48^{b}$ & 145.15 & 19.580 & 0.039 & 0.009 & 0.402 & 0.954 \\
\hline Chao1 & $279.94^{a}$ & $180.82^{a b}$ & $136.95^{b}$ & 137.36 & 20.244 & 0.041 & 0.008 & 0.367 & 0.993 \\
\hline \multicolumn{10}{|l|}{42 days old } \\
\hline Observed_species & 432 & 334 & 410 & 571 & 37.701 & 0.652 & 0.178 & 0.029 & 0.123 \\
\hline Shannon & 2.72 & 3.14 & 3.52 & 4.11 & 0.240 & 0.533 & 0.048 & 0.154 & 0.376 \\
\hline Simpson & 0.58 & 0.69 & 0.80 & 0.85 & 0.041 & 0.183 & 0.015 & 0.124 & 0.644 \\
\hline Ace & 483.00 & 387.50 & 458.50 & 624.45 & 39.969 & 0.714 & 0.205 & 0.042 & 0.141 \\
\hline Chao1 & 475.80 & 367.40 & 458.70 & 624.81 & 39.534 & 0.604 & 0.167 & 0.024 & 0.126 \\
\hline
\end{tabular}

a,b Means $(n=5)$ within a row with different letters differed significantly $(P<0.05)$; SEM = standard error of the mean.

$\mathrm{CON}=$ the control treatment, $\mathrm{LD}=$ the low dose of dandelion treatment, $\mathrm{HD}=$ the high dose of dandelion treatment, $\mathrm{CTC}=$ the chlortetracycline treatment. ANONA = the treatment effect of CON, LD and HD; CON vs CTC/ LD vs CTC/ HD vs CTC = contrasts comparison of the chlortetracycline group with groups of different additive doses of dandelion.

The effects of dandelion on the relative abundances of ileal microbiota at the phyla level are shown in Table 4. Firmicutes was the dominant phylum in the ileal microbiota at 21 and 42 days old. At 21 days old, compared to the CON group, the relative abundance of Firmicutes was increased by adding two doses of dandelion and the relative abundance of Bacteroidetes was reduced by adding two doses of dandelion and CTC to the diet $(P<0.05)$. At 42 days old, there was no significant difference in the relative abundance of ileal bacteria between dandelion treatment and CON $(P>0.05)$. Compared to CTC, the relative abundance of Actinobacteriota in the ileum of CON, LD, and HD birds was increased $(P<0.05)$. 
Table 4

Effects of dandelion on the relative abundances of ileal microbiota at the phyla level (\%)

\begin{tabular}{|c|c|c|c|c|c|c|c|c|c|}
\hline \multirow[t]{2}{*}{ Items } & \multicolumn{4}{|c|}{ Treatment group } & \multirow[t]{2}{*}{ SEM } & \multicolumn{4}{|l|}{$P$-value } \\
\hline & CON & LD & HD & СТC & & ANONA & $\begin{array}{l}\text { CONvs } \\
\text { CTC }\end{array}$ & $\begin{array}{l}\text { LD vs } \\
\text { CTC }\end{array}$ & $\begin{array}{l}\text { HD vs } \\
\text { CTC }\end{array}$ \\
\hline \multicolumn{10}{|l|}{21 days old } \\
\hline Firmicutes & $74.26^{b}$ & $90.57^{a}$ & $92.29^{a}$ & 87.57 & 2.687 & 0.030 & 0.061 & 0.656 & 0.485 \\
\hline Cyanobacteria & 11.06 & 6.36 & 4.86 & 8.28 & 1.574 & 0.288 & 0.551 & 0.680 & 0.465 \\
\hline Proteobacteria & 3.25 & 1.91 & 2.55 & 3.49 & 0.484 & 0.662 & 0.867 & 0.283 & 0.519 \\
\hline Bacteroidetes & $9.01^{a}$ & $0.82^{b}$ & $0.13^{b}$ & 0.22 & 1.169 & 0.015 & 0.002 & 0.808 & 0.973 \\
\hline \multicolumn{10}{|l|}{42 days old } \\
\hline Firmicutes & 86.04 & 90.62 & 73.78 & 81.47 & 3.352 & 0.234 & 0.631 & 0.342 & 0.423 \\
\hline Cyanobacteria & 4.99 & 0.83 & 14.15 & 10.73 & 2.471 & 0.128 & 0.404 & 0.159 & 0.616 \\
\hline Proteobacteria & 4.74 & 6.28 & 9.54 & 3.67 & 1.286 & 0.520 & 0.772 & 0.485 & 0.126 \\
\hline Bacteroidetes & 3.12 & 1.19 & 1.60 & 2.08 & 0.530 & 0.528 & 0.513 & 0.576 & 0.762 \\
\hline Actinobacteriota & 0.33 & 0.40 & 0.35 & 1.17 & 0.131 & 0.904 & 0.019 & 0.029 & 0.021 \\
\hline \multicolumn{10}{|c|}{ Only bacteria with a relative abundance greater than $1 \%$ are shown in the table. } \\
\hline \multicolumn{10}{|c|}{$\begin{array}{l}a, b \text { Means }(n=5) \text { within a row with different letters differed significantly }(P<0.05) \text {; SEM = standard error of the } \\
\text { mean. }\end{array}$} \\
\hline \multicolumn{10}{|c|}{$\begin{array}{l}C O N=\text { the control treatment, } L D=\text { the low dose of dandelion treatment, } H D=\text { the high dose of dandelion } \\
\text { treatment, } C T C=\text { the chlortetracycline treatment. ANONA the treatment effect of } C O N \text {, } L D \text { and } H D ; C O N \text { V } C T C \text { / } \\
L D \text { VS CTC/ HD vS } C T C=\text { contrasts comparison of the chlortetracycline group with groups of different additive } \\
\text { doses of dandelion. }\end{array}$} \\
\hline
\end{tabular}

Table 5 shows the relative abundance of ileal microbiota at the genera level. As reported in Table 7, Lactobacillus was the dominant genus in the ileal microbiota, belonging to Firmicutes. Consistent with the phylum level results, at 21 days old, the relative abundance of Lactobacillus in the CON group was lower than that in the LD and HD groups $(P<0.05)$, the relative abundance of Bacteroides in the CON group was higher than that in the LD, HD, and CTC groups $(P<0.05)$. At 42 days old, the relative abundance of Lactobacillus in the LD group was higher than that in the CTC group $(P<0.05)$, the relative abundance of Lysinibacillus in the CON and LD groups was lower than that in the CTC group $(P<0.05)$, while there was no significant difference in other microbiota $(P>0.05)$. 
Table 5

Effects of dandelion on the relative abundances of ileal microbiota at the genera level (\%)

\begin{tabular}{|c|c|c|c|c|c|c|c|c|c|}
\hline \multirow[t]{2}{*}{ Items } & \multicolumn{4}{|c|}{ Treatment group } & \multirow[t]{2}{*}{ SEM } & \multicolumn{4}{|l|}{ P-value } \\
\hline & CON & LD & HD & стC & & ANONA & $\begin{array}{l}\text { CON } \\
\text { vs } \\
\text { CTC }\end{array}$ & $\begin{array}{l}\text { LD vs } \\
\text { CTC }\end{array}$ & $\begin{array}{l}\text { HD vs } \\
\text { CTC }\end{array}$ \\
\hline \multicolumn{10}{|l|}{21 days old } \\
\hline Lactobacillus & $39.21^{b}$ & $64.21^{\mathrm{a}}$ & $80.89^{a}$ & 62.68 & 5.071 & 0.004 & 0.060 & 0.897 & 0.135 \\
\hline Romboutsia & 5.79 & 9.05 & 3.76 & 9.76 & 1.793 & 0.472 & 0.461 & 0.895 & 0.270 \\
\hline CandidatusArthromitus & 8.99 & 8.46 & 3.50 & 5.67 & 1.352 & 0.409 & 0.402 & 0.480 & 0.582 \\
\hline unidentifiedChloroplast & 11.06 & 6.36 & 4.86 & 8.28 & 1.574 & 0.288 & 0.551 & 0.680 & 0.465 \\
\hline EscherichiaShigella & 0.94 & 0.66 & 1.14 & 1.54 & 0.270 & 0.740 & 0.465 & 0.289 & 0.625 \\
\hline Enterococcus & 2.81 & 2.57 & 1.11 & 2.14 & 0.499 & 0.531 & 0.655 & 0.775 & 0.495 \\
\hline Bacteroides & $5.73^{\mathrm{a}}$ & $0.67^{b}$ & $0.07^{b}$ & 0.15 & 0.783 & 0.029 & 0.005 & 0.770 & 0.962 \\
\hline Streptococcus & 2.90 & 0.93 & 0.83 & 1.28 & 0.626 & 0.540 & 0.394 & 0.851 & 0.809 \\
\hline \multicolumn{10}{|l|}{42 days old } \\
\hline Lactobacillus & 80.52 & 83.70 & 63.77 & 61.48 & 4.053 & 0.213 & 0.085 & 0.048 & 0.828 \\
\hline unidentifiedChloroplast & 4.97 & 0.62 & 14.14 & 10.71 & 2.478 & 0.123 & 0.404 & 0.152 & 0.616 \\
\hline EscherichiaShigella & 3.28 & 0.81 & 6.00 & 1.03 & 1.123 & 0.384 & 0.483 & 0.944 & 0.132 \\
\hline Pseudomonas & 0.58 & 1.85 & 2.13 & 1.11 & 0.318 & 0.275 & 0.555 & 0.407 & 0.262 \\
\hline Bacteroides & 1.88 & 0.37 & 0.42 & 0.68 & 0.347 & 0.341 & 0.239 & 0.758 & 0.796 \\
\hline CandidatusArthromitus & 0.46 & 1.44 & 0.75 & 1.28 & 0.250 & 0.415 & 0.274 & 0.825 & 0.478 \\
\hline Lysinibacillus & 0.07 & 0.10 & 0.87 & 2.61 & 0.357 & 0.285 & 0.007 & 0.008 & 0.051 \\
\hline \multicolumn{10}{|c|}{ Only bacteria with a relative abundance greater than $1 \%$ are shown in the table. } \\
\hline \multicolumn{10}{|c|}{$\begin{array}{l}a, b \text { Means }(n=5) \text { within a row with different letters differed significantly }(P<0.05) \text {; SEM = standard error of the } \\
\text { mean. }\end{array}$} \\
\hline
\end{tabular}


Table 6

Effects of dandelion on the short-chain fatty acid concentrations in the ileal content of broilers $(\mu \mathrm{g} / \mathrm{g})$

\begin{tabular}{|c|c|c|c|c|c|c|c|c|c|}
\hline \multirow[t]{2}{*}{ Items } & \multicolumn{4}{|c|}{ Treatment group } & \multirow[t]{2}{*}{ SEM } & \multicolumn{4}{|l|}{ P-value } \\
\hline & CON & LD & HD & CTC & & ANONA & $\begin{array}{l}\text { CON vs } \\
\text { CTC }\end{array}$ & $\begin{array}{l}\text { LD vs } \\
\text { CTC }\end{array}$ & $\begin{array}{l}\text { HD vs } \\
\text { CTC }\end{array}$ \\
\hline \multicolumn{10}{|l|}{21 days old } \\
\hline Acetic acid & 8.026 & 7.783 & 6.175 & 5.035 & 0.9523 & 0.797 & 0.300 & 0.317 & 0.689 \\
\hline $\begin{array}{l}\text { Propionic } \\
\text { acid }\end{array}$ & 0.367 & 0.401 & 0.251 & 0.994 & 0.1252 & 0.844 & 0.058 & 0.051 & 0.029 \\
\hline Butyric acid & 0.168 & 0.252 & 0.146 & 0.283 & 0.0182 & 0.082 & 0.011 & 0.453 & 0.005 \\
\hline Total SCFA & 8.414 & 9.593 & 7.947 & 6.312 & 1.0000 & 0.856 & 0.468 & 0.263 & 0.595 \\
\hline \multicolumn{10}{|l|}{42 days old } \\
\hline Acetic acid & 7.226 & 9.024 & 4.261 & 6.660 & 1.0261 & 0.327 & 0.848 & 0.428 & 0.421 \\
\hline $\begin{array}{l}\text { Propionic } \\
\text { acid }\end{array}$ & 0.301 & 0.510 & 0.123 & 0.106 & 0.0825 & 0.350 & 0.412 & 0.084 & 0.938 \\
\hline Butyric acid & 0.053 & 0.094 & 0.182 & 0.052 & 0.0355 & 0.559 & 0.990 & 0.677 & 0.211 \\
\hline Caproic acid & 0.057 & 0.047 & 0.030 & 0.031 & 0.0046 & 0.108 & 0.042 & 0.168 & 0.896 \\
\hline Total SCFA & 8.837 & 9.676 & 4.590 & 6.849 & 1.1116 & 0.288 & 0.543 & 0.368 & 0.470 \\
\hline \multicolumn{10}{|c|}{$\begin{array}{l}\text { a,b Means }(n=5) \text { within a row with different letters differed significantly }(P<0.05) \text {; SEM = standard error of the } \\
\text { mean. }\end{array}$} \\
\hline \multicolumn{10}{|c|}{$\begin{array}{l}C O N=\text { the control treatment, } L D=\text { the low dose of dandelion treatment, } H D=\text { the high dose of dandelion } \\
\text { treatment, } C T C=\text { the chlortetracycline treatment. ANONA = the treatment effect of CON, LD and HD; CON vs CTC/ } \\
\text { LD vS CTC/ HD vS CTC = contrasts comparison of the chlortetracycline group with groups of different additive } \\
\text { doses of dandelion. }\end{array}$} \\
\hline
\end{tabular}


Table 7

Dietary composition and nutrient levels of basal diets.

Items
Grower phase

(d 22 to 42)

Ingredient (\%)

Corn

52.50

58.80

Soybean meal

40.00

33.80

Soybean oil

3.00

3.00

Dicalcium phosphate

1.90

1.80

Limestone

1.08

1.22

Salt

0.37

0.37

Lysine

0.05

0.03

Methionine

0.19

0.07

Premix $^{1}$

0.80

0.80

Choline

0.11

0.11

Total

100.00

100.00

Nutrient levels ${ }^{2}$

Metabolic energy ( $\mathrm{MJ} / \mathrm{kg}$ )

12.42

12.62

Crude protein (\%)

22.27

19.24

Ether extract (\%)

4.69

5.45

Crude fiber (\%)

3.50

3.26

Ca (\%)

0.99

1.20

Total phosphorus (\%)

0.59

0.67

Non-phytate phosphorus (\%)

0.54

0.57

${ }^{1}$ The premix provided the following per kg of diet: VA, $9000 \mathrm{IU} ; \mathrm{VD}_{3}, 3000 \mathrm{IU} ; \mathrm{VE}, 26 \mathrm{mg} ; \mathrm{VK}_{3}, 1.20 \mathrm{mg} ; \mathrm{VB}_{1}, 3.00$ $\mathrm{mg} ; \mathrm{VB}_{2}, 8.00 \mathrm{mg} ; \mathrm{VB}_{6}, 4.40 \mathrm{mg} ; \mathrm{VB}_{12}, 0.012 \mathrm{mg}$; nicotinic acid, $45 \mathrm{mg}$; folic acid, $0.75 \mathrm{mg}$; biotin, $0.20 \mathrm{mg}$; choline, 1100 mg; D-pantothenic acid, 15 mg; Fe, 100 mg; Cu, 10 mg; Zn, 108 mg; Mn, 120 mg; I 1.5 mg; Se, 0.35 mg.

${ }^{2}$ Metabolic energy is a calculated value, while the others are measured values.

\section{SCFA content of broilers following different treatments}

Table 6 shows that acetic acid is the most abundant SCFA in the ileum in all groups. At 21 days old, compared to CTC birds, the propionic acid content of HD birds and the butyric acid content of CON and HD decreased $(P<0.05)$. At 42 days old, there was no significant difference between LD, HD, and CON groups in each SCFA $(P>0.05)$. Furthermore, the content of caproic acid in the CON birds was higher than that in the CTC birds $(P<0.05)$ 


\section{Discussion}

$\mathrm{CH}$ such as dandelion have received widespread attention for their beneficial uses, including promoting growth and improving immunity and intestinal microbiota $[24,25]$. $\mathrm{CH}$ have demonstrated positive effects on growth performance, such as increasing ADG and decreasing ADFI [10]. It is accepted that feed costs represent approximately about $70 \%$ of the cost of poultry production, making a bird's ability to use feed efficiently particularly important [26]. Therefore, having a lower F/G is of great significance for poultry production. Numerous studies have reported that dietary supplementation with $\mathrm{CH}$ can enhance the growth performance of broilers, indeed, dandelion leaf powder and licorice essential oil have been shown to have a positive effect on improving the growth performance, in which $2.0 \mathrm{~g} / \mathrm{kg}$ dandelion leaf powder treatments obtained the best feed conversion factor, and 200 $\mathrm{mg} / \mathrm{kg}$ licorice essential oil showed greater weight gain and better feed conversion ratio compared to diets containing $400 \mathrm{mg} \mathrm{LEO} / \mathrm{kg}$ [27]. Similar to the above results, our study showed that dandelion is beneficial for improving the growth performance of broilers at both the starter and overall phases, as indicated by the decreased $\mathrm{ADFI}$ and $\mathrm{F} / \mathrm{G}$. This result demonstrated that diets supplemented with dandelion could enhance feed utilization rate of broilers, thus reducing some feed costs during production. The improved growth performance of broilers in the current study may be related to the better digestion and absorption function, possibly due to the beneficial changes in intestinal barrier function regulated by tight junction proteins, intestinal immunity and intestinal microbiota [28, 29].

The physical, chemical, immunological and microbiological barriers in the intestinal tract constitute the complete intestinal barrier, which plays an important defensive role against the invasion of harmful substances from the intestines [8]. Research has shown that the intestinal barrier can prevent the invasion of pathogens and food-borne antigens through the interaction between the four barriers, thereby regulating the homeostasis of the intestinal environment, which is essential for maintaining the homeostasis of health and disease [30]. Therefore, a study on intestinal barrier function is crucial for the continued success of poultry production.

The physical barrier is a tightly connected intestinal epithelial structure, which is composed of intestinal mucosal epithelial cells and their tight junctions [31]. Claudins, occludin and zonula occludens are the main proteins forming tight junctions, and the dysfunction of these proteins is harmful to the physical barrier function [32]. Increasing evidence indicates that $\mathrm{CH}$ improves the physical barrier by enhancing the expression of tight junction proteins [33, 34]. Consistently, this research showed that broilers fed a dandelion-supplemented diet exhibited significant upregulation of claudin and occludin-1 gene expression in the ileum at 21 days old compared to CON and CTC birds. Moreover, the gene expression of ZO-1 in HD birds was significantly increased at 21 days old. At 42 days old, the gene expression of claudin and occludin-1 in LD and ZO-1 in HD were significantly increased, resulting in improved physical barrier function. Tan et al. found that dandelion extract significantly increased intestinal ZO-1 and occludin mRNA levels [21]. Therefore, dandelion can improve physical barrier function by increasing various tight junction proteins, which may eventually promote the integrity of the intestinal barrier.

The chemical barrier is mainly composed of mucus covering the intestinal epithelium, including digestive fluid, lysozyme, mucin and other substances in mucus. Mucins are secreted by intestinal goblet cells, which are an important component of the intestinal chemical barrier and are of great significance to the integrity of the intestinal barrier [35, 36]. Mucins include secreted mucins [mucin-2, mucin-5AC, mucin-5B, mucin-6 and so on] and cell surface mucins [MUC1, mucin-3A, mucin-3B, mucin-4, mucin-12], in which cell surface mucins must prevent enteric pathogens from penetrating the inner mucus layer, to protect the mucosa from infection [37]. In this study, dietary supplementation with two dose of dandelion both enhanced the gene expression of MUC1 at 21 days old, while 500

Page $11 / 26$ 
$\mathrm{mg} / \mathrm{kg}$ dandelion increased the gene expression of MUC1 at 42 days old, which resulted in better chemical barrier function and promote the integrity of the intestinal barrier.

The intestinal immunological barrier is mainly composed of gut-associated lymphatic tissues. SlgA is the primary component of the immunological barrier and acts as the first line of defense of the intestinal lumen from antigens and pathogens by preventing their access to epithelial cell receptors [38, 39]. In addition, the equilibrium among proand anti-inflammatory cytokines is necessary for gut immunological homeostasis [22]. However, in contrast to previous studies, we observed no effects of dandelion on inflammatory cytokines and SIgA in the current study [40, 41], indicating that dandelion failed to induce any notable effects in the immunological barrier.

The microbiological barrier is a microecosystem formed by intestinal microbial flora in the intestinal cavity environment, which is an important component of the intestinal barrier [42]. The intestinal microbiota has coevolved with the host to form a stable intestinal micro-environment, which provides the host a broad range of biofunctions, such as inhibiting pathogen infection, promoting nutritional metabolism, regulating the immune response, and improving the barrier function of the body $[43,44]$. Alpha diversity is used to analyze the complexity of species diversity and beta diversity is to evaluate differences of species complexity among different treatment [45]. $\mathrm{CH}$ have been shown to affect the alpha diversity indices such as increasing Shannon index and decreased Simpson index, indicating that $\mathrm{CH}$ can improve the diversity of intestinal microbiota [46]. However, in contrast to previous studies, our results showed that dandelion decreased the diversity of intestinal microbiota, which may be due to the antibacterial activity of dandelion $[47,48]$. The results of PCoA shown that these two doses of dandelion have no effects on beta diversity, which difference with the result of Tan et al. [20] in golden pompano Trachinotus ovatus. This may indicate that dandelion has little effect on beta diversity in broilers.

In this study, dandelion enhanced the relative abundance of Firmicutes, decreased the relative abundance of Bacteroidetes at the phyla level, increased the relative abundance of Lactobacillus, and decreased the relative abundance of Bacteroides at the genera level at 21 days old. Consistent with the findings of a previous study, the abundance of Firmicutes was gradually increased while the abundance of Bacteroidetes was decreased [49]. This may indicate that Firmicutes is a more effective energy source than Bacteroidetes, thus promoting more efficient absorption of carbohydrate and increased weight gain. Overall, dandelion was beneficial for improving the feed utilization efficiency and the improved growth performance of broilers; this may be mediated by the change in the relative abundance of Firmicutes and Bacteroidetes. However, at 42 days old, dandelion had no significant effect on the intestinal microbiota at the levels of phyla and genera. Moreover, the relative abundance of Escherichia-Shigella in the LD group was the lowest at the genus level, indicating that a sufficient concentration of dandelion could decrease harmful microbiota and improve intestinal microbiota structure. The above results showed that dandelion could improve the structure of the intestinal microbiota, although the appropriate concentration needs to be determined. SCFAs are the main metabolic end products of intestinal microbiota, which are not only an important energy source for the gut microbiota itself and intestinal epithelial cells, but also play important roles in balancing intestinal homeostasis, and inhibiting growth and colonization of pathogens [50]. Butyric acid, as the main energy material of epithelial cells, is mainly produced by the metabolism of Firmicutes [51]. Butyric acid affects the proliferation and differentiation of intestinal epithelial cells, which helps to strengthen intestinal tight junctions [52]. This study showed that dandelion was increased the butyric acid content to a certain extent, which may lead to the enhancement of tight junction connections.

\section{Conclusions}

Page $12 / 26$ 
The current study demonstrated that dandelion decreased ADFI and F/G; increased the gene expression of claudin, occludin-1, ZO-1, and MUC1; and enhanced the relative abundance of Firmicutes and decreased the relative abundance of Bacteroidota. Dandelion has a potential beneficial effect on growth performance by improving the intestinal barrier and may thus represent an effective antibiotic alternative in poultry production.

\section{Methods}

\section{Ethics statement}

The ethics of the experimental protocol were approved by Inner Mongolia Agricultural University Research Ethics Committee (permission number of [2020]065). All animal experiments were performed following the ARRIVE guidelines, and in accordance with national guidelines and regulations (GB 14925 - 2010) for reporting animal research as much as possible. The dandelion used in this experiment was purchased from Bozhou zhenshanyuan traditional Chinese medicine Sales Co., Ltd (Anhui Province, China). According to their instructions, the dandelion was cultivated in Bozhou in Northern Anhui Province of China, and the cultivation procedure was performed in compliance with the Convention on Biological Diversity and the Convention on the Trade and Endangered Species of Wild Fauna and Flora. The samples of dandelion are preserved in the Inner Mongolia Herbivorous Livestock Feed Engineering Technology Research Center, Hohhot, China.

\section{Animal, experimental design and diets}

The study was carried out in compliance with the ARRIVE guidelines and national guidelines and regulations (GB 14925 - 2010). One-hundred and sixty 1-day-old AA male broilers (purchased from Inner Mongolia Yuean breeding Co., Ltd) with similar birth weights and good physical condition were randomly divided into four groups as

follows:1) Control group was provided with a corn-soybean meal basal diet (CON); 2) Low dose group was provided with a basal diet supplemented with $500 \mathrm{mg} / \mathrm{kg}$ dandelions (LD); 3) High dose group was provided with a basal diet supplemented with $1000 \mathrm{mg} / \mathrm{kg}$ dandelions (HD); 4) Positive control group was provided with a basal diet supplemented with $50 \mathrm{mg} / \mathrm{kg}$ chlortetracycline (CTC). Each group comprised five replicates with eight chickens per replicate. The experimental period was 42 days, which consisted of two phases: a starter phase (d 1 to 21 ) and a grower phase ( $\mathrm{d} 22$ to 42 ). The whole parts of dandelion were dried, grounded, and added to the basal diets. All basal diets were formulated to meet the nutrient recommendations of Feeding Standard of Chicken, China (NY/T 33-2004) (Chinese Ministry of Agriculture, 2004). The compositions of the basal diets and the nutrient levels for starter and grower phases are presented in Table 7.

Chickens had free access to feed and water throughout the trial period. The initial temperature of the room was set at $33-35^{\circ} \mathrm{C}$ and reduced by $2-3^{\circ} \mathrm{C}$ weekly until $23^{\circ} \mathrm{C}$, at which point the temperature was maintained until the end of the experiment. The lighting schedule was $24 \mathrm{~L}$ ( $24 \mathrm{~h}$ of lighting and $0 \mathrm{~h}$ of dark per day) for $\mathrm{d} 1-3 ; 16 \mathrm{~L}$ : 8D d 414, and natural light after $\mathrm{d} 14$.

\section{Sample collection}

At $21 \mathrm{~d}$ and $42 \mathrm{~d}$, one bird was randomly selected from each cage for sampling. The chicken was dissected to collect ileal tissue and ileal digesta and store in cryopreservation tubes. These samples were immediately frozen in liquid nitrogen and stored at $-80^{\circ} \mathrm{C}$ until analysis.

\section{Growth performance}


The birds were fasted for $12 \mathrm{~h}$ before weighting. At the end of each week during the experimental period, birds were weighed, and their weights were recorded. The feed intake of the experimental birds during the experimental period was counted by repetition. The average daily gain (ADG), average daily feed intake (ADFI), and feed to gain ratio $(F / G)$ were calculated for each period and for the overall experiment.

\section{Gene expression of tight junction proteins and mucin in ileum}

Total RNA extraction was performed using TRIzol reagent according to the manufacturer's instructions. The RNA concentration was determined by agarose gel electrophoresis, and the purity was determined by comparing the A260/A280 ratio using a spectrophotometer (NanoDrop ND-2000). Subsequently, the total RNA from each sample was reversely transcribed to $c D N A$ using PrimeScript ${ }^{\mathrm{TM}} \mathrm{RT}$ reagent Kit with gDNA Eraser. The synthetic cDNA was stored at $-20{ }^{\circ} \mathrm{C}$ until Real-time fluorescent quantitative PCR (RT qPCR) analysis. RT qPCR was performed in 96 well microplates using SYBR Premix Ex Taq $\otimes$. The specific sequences of primers are listed in Table 8. The relative mRNA abundance of the target genes was calculated using the $2^{-\triangle \triangle C T}$ method with an endogenous reference gene ( $\beta$ actin) [22].

Table 8

Gene-specific primers of broilers.

\begin{tabular}{|c|c|c|}
\hline \multicolumn{3}{|c|}{ Gene-specifıc primers of brollers. } \\
\hline Gene & Primer sequences $\left(5^{\prime} \rightarrow 3^{\prime}\right)$ & Product size (bp) \\
\hline \multirow[t]{2}{*}{ Claudin } & F:CCGCTGTCCTGAGCAGAGTT & \multirow[t]{2}{*}{161} \\
\hline & R:TTTCCAGTGGCGATACCTAC & \\
\hline \multirow[t]{2}{*}{ Occludin-1 } & F:GGTTCCTCATCGTCATCCTG & \multirow[t]{2}{*}{149} \\
\hline & R:TTCTTCAСССАСТССТССАС & \\
\hline \multirow[t]{2}{*}{ ZO-1 } & F:CTAAGGGGAAGCCAACTGAT & \multirow[t]{2}{*}{215} \\
\hline & R:ATTCTGAGGTGGAGGAGGGT & \\
\hline \multirow[t]{2}{*}{ MUC1 } & F:ATATCCGTGCCGCTGGTT & \multirow[t]{2}{*}{239} \\
\hline & R:GCCGGGCGTTGTTAATGT & \\
\hline
\end{tabular}

\section{Determination of immunity in ileum}

Ileal tissue samples stored at $-80^{\circ} \mathrm{C}$ were removed for homogenization, and the supernatant was obtained by centrifugation at $3500 \mathrm{r} / \mathrm{min}$ for $10 \mathrm{~min}$ in a low-temperature high-speed centrifuge. The concentration of Interleukin (IL)-1, IL-2, IL-10, secretory immunoglobulin A (SIgA), and tumor necrosis factor-a (TNF-a) in the supernatant were measured using commercial ELISA kits, and the assay procedure was strictly performed according to the instructions.

\section{lleal microbiota analysis}

Total bacterial DNA was extracted from the ileal digesta sample using the QIAamp DNA Stool Mini Kit (QIAGEN, Germany) according to the manufacturer's protocols. Briefly, the bacterial 16S rRNA gene spanning the V4 hypervariable regions was amplified using universal primers with a PCR system. Amplicon libraries for all samples were quantified by Qubit 2.0 Fluorometer (Thermo Fisher Scientific, Waltham, US) and sequenced on an NovaSeq6000 platform by Novogene Bioinformatics Technology Co., Ltd. (Beijing, China) to generate $2 \times 250 \mathrm{bp}$ paired-end reads. 
According to the barcode sequence and the PCR amplified primer sequence, sample data were separated from the off-line data. After intercepting the barcode and primer sequence, FLASH was used (v1.2.7, http://ccb.jhu.edu/software/FLASH/). The reads of each sample were spliced, where the spliced sequence represents the original tag data (Raw tags). The Raw tags obtained by splicing were strictly filtered to obtain highquality tag data (Clean tags). Refer to qiime (v1.9.1, http://qiime.org/scripts/split_libraries_fastq.html)Tags quality control process: a) Tags interception: cut Raw tags from the first low-quality base site with continuous low-quality value (default quality threshold is $<=19$ ) and base number reaching the set length (default length value is 3 ); $b$ ) Tags length filtering: the Tags data set obtained by intercepting Tags further filters out Tags in which the continuous high-quality base length is less than $75 \%$ of the Tags length. The Tags obtained after the above processing need to be processed to remove the chimera sequence, and the Tags sequence passes (https://github.com/torognes/vsearch/) compare with the species annotation database to detect chimeric sequences, and finally remove the chimeric sequences to obtain the final effective Tags (Effective Tags). The similarity of sequences at $97 \%$ was defined as an operational taxonomic unit (OTU). The representative OTUs sequences were annotated using RDP classifier (V 2.2) and Greengenes database (V 13.5). The data from all of the ileal digesta samples were then normalized for the analysis of alpha and beta diversity. The various alpha diversity indexes, including Observed_species, Shannon, Chao1, ACE, and Simpson index, were used. Weighted UniFrac distance metrics were used to generate principal coordinate analysis (PCoA) plot.

\section{Short chain fatty acids (SCFA) analysis}

The concentration of SCFA (acetic acid, propionic acid, butyric acid, and caproic acid) was measured using liquid chromatography as described by Shivatare et al. (23). The column was a BEH C8 $(100 \times 2.1 \mathrm{~mm}, 1.7 \mu \mathrm{m})$ column and the mobile phase was composed of water (including $0.1 \%$ formic acid) (A) and methanol (B). The sample injection volume was $4 \mu \mathrm{l}$ and the flow rate was maintained at $0.3 \mathrm{~mL} \mathrm{~min}^{-1}$. The chromatographic peaks of SCFAs were confirmed by comparing their retention time with the reference standard.

\section{Statistical analysis}

Experiment data were collated and preliminary processed using Excel software. Data from CON, LD and HD treatments were analyzed by one-way analysis of variance (ANONA) with Duncan's multiple comparisons using SAS 9.2 statistical software. CTC was also compared pairwise with CON, LD and HD treatments using specific preplanned contrasts. Differences were considered significant at $\mathrm{P}<0.05$.

\section{Abbreviations}

$\mathrm{CH}$

Chinese herbs

CON

Control group

LD

Low dose group

HD

High dose group

CTC

Positive control group

ADG 
Average daily gain

ADFI

Average daily feed intake

F/G

feed to gain ratio

IL

Interleukin

$\operatorname{SIg} A$

Secretory immunoglobulin A

TNF-a

tumor necrosis factor-a

PCoA

Principal coordinate analysis

SCFA

Short chain fatty acids

ANONA

One-way analysis of variance

ZO-1

Zonula occludens-1

MUC1

Mucin-1.

\section{Declarations}

\section{Ethics approval and consent to participate}

The ethics of the experimental protocol were approved by Inner Mongolia Agricultural University Research Ethics Committee (permission number of [2020]065). All animal experiments were performed following the ARRIVE guidelines, and in accordance with national guidelines and regulations (GB 14925-2010) for reporting animal research as much as possible. The dandelion used in this experiment was purchased from Bozhou zhenshanyuan traditional Chinese medicine Sales Co., Ltd (Anhui Province, China). According to their instructions, the dandelion was cultivated in Bozhou in Northern Anhui Province of China, and the cultivation procedure was performed in compliance with the Convention on Biological Diversity and the Convention on the Trade and Endangered Species of Wild Fauna and Flora. The samples of dandelion are preserved in the Inner Mongolia Herbivorous Livestock Feed Engineering Technology Research Center, Hohhot, China.

\section{Consent for publication}

Not applicable.

\section{Availability of data and materials}

The datasets used and/or analysed during the current study are available from SRA NCBI repository under the Bioproject accession number (PRJNA808831).

\section{Competing interests}


The authors declare that they have no competing interests.

\section{Funding}

This study was supported by the Major Science and Technology Program of Inner Mongolia Autonomous Region (2021ZD0024-4, 2021ZD0023-3, 2020ZD0004), and the Key Technology Project of Inner Mongolia Autonomous Region (2020GG0030).

\section{Authors' contributions}

JM: conceptualization, methodology, investigation, formal analysis, data curation, and writing - original draft. YW: conceptualization, methodology, investigation, formal analysis, writing - review \& editing, and funding acquisition. WW: formal analysis, data curation, and writing - review \& editing. TD: resources, investigation, and data curation. NY, TG, HG, and NL: resources, and investigation. XA: conceptualization, methodology, and funding acquisition. JQ: supervision, project administration, and funding acquisition.

\section{Acknowledgements}

Not applicable.

\section{Author details}

${ }^{1}$ College of Animal Science, Inner Mongolia Agricultural University, Hohhot 010018, China; ${ }^{2}$ Inner Mongolia Herbivorous Livestock Feed Engineering Technology Research Center, Hohhot 010018, China; ${ }^{3}$ Key Laboratory of Smart Animal Husbandry at Universities of Inner Mongolia Automomous Region, Hohhot 010018, China.

\section{References}

1. Heckert RA, Estevez I, Russek-Cohen E, Pettit-Riley R. Effects of density and perch availability on the immune status of broilers. Poult Sci. 2002;81(4):451.

2. Relić R, Sossidou E, Dedousi A, Peri L, Uki-Stoji M. Behavioral and health problems of poultry related to rearing systems. Ankara Üniv Vet Fak Derg. 2019;66(4):423-428.

3. Low CX, Tan TH, Mutalib NSA, Pusparajah P, Lee LH. Unveiling the Impact of Antibiotics and Alternative Methods for Animal Husbandry: A Review. Antibiotics. 2021;10(5): 578.

4. Oso AO, Suganthi RU, Reddy GBM, Malik PK, Thirumalaisamy G, Awachat VB, et al. Effect of dietary supplementation with phytogenic blend on growth performance, apparent ileal digestibility of nutrients, intestinal morphology, and cecal microflora of broiler chickens. Poult Sci. 2019,98(10):4755-4766.

5. Zhang S, Chen DC. Facing a new challenge: the adverse effects of antibiotics on gut microbiota and host immunity. Chin Med J (Beijing, China, Engl. Ed.). 2019;132(10):1135.

6. Yang H, Paruch L, Chen X, Eerde AV, Clarke JL. Antibiotic application and resistance in swine production in china: current situation and future perspectives. Front Vet Sci. 2019;6:136.

7. Wang B, Gong L, Zhou Y, Tang L, Li W. Probiotic Paenibacillus polymyxa 10 and Lactobacillus plantarum 16 enhance growth performance of broilers by improving the intestinal health. Anim Nutr. 2021;7(3):829-840.

8. Cui Y, Wang Q, Chang R, Zhou X, Xu C. Intestinal barrier function-nafld interactions and possible role of gut microbiota. J Agric Food Chem. 2019. 
9. Anderson RC, Dalziel J E, Gopal PK, Bassett S, Ellis A, Roy N C. The Role of Intestinal Barrier Function in Early Life in the Development of Colitis. InTech. 2012.

10. Gong J, Yin F, Hou Y, Yin Y. Review: chinese herbs as alternatives to antibiotics in feed for swine and poultry production: potential and challenges in application. Can J Anim Sci. 2014;94(2):223-241.

11. Kong XF, Wu GY, Liao YP, Hou ZP, Liu HJ, Yin FG. Effects of Chinese herbal ultra-fine powder as a dietary additive on growth performance, serum metabolites and intestinal health in early-weaned piglets. Livest Sci. 2007;108(1-3): 272-275.

12. Song Z, Cheng K, Zhang L, Wang T. Dietary supplementation of enzymatically treated Artemisia annua could alleviate the intestinal inflammatory response in heat-stressed broilers. J Therm Biol. 2020;98(1):skz396.

13. Yang L, Liu G, Lian KX, Qiao YJ, Zhang BJ, Zhu XQ, et al. Dietary leonurine hydrochloride supplementation attenuates lipopolysaccharide challenge-induced intestinal inflammation and barrier dysfunction by inhibiting the NF-KB/MAPK signalling pathway in broilers. J Anim Sci. 2019;97(4):1679-1692.

14. Zhao X, Li L, Luo Q, Ye M, Luo G, Kuang Z. Effects of mulberry (morus alba I.) leaf polysaccharides on growth performance, diarrhea, blood parameters, and gut microbiota of early-weanling pigs. Livest Sci. 2015;177:8894.

15. Peng MF, Fang XY, Su-Hui WU, Miao MS. Application and Analysis of Chinese Patent Medicines Containing Dandelion. DEStech Transactions on Environment, Energy and Earth Sciences. 2017.

16. González-Castejón M, Visioli F, Rodriguez-Casado A. Diverse biological activities of dandelion. Nutr Rev. 2013;70:534-547.

17. Jeon HJ, Kang HJ, Jung HJ, Kang YS, Lim CJ, Kim YM. Anti-inflammatory activity of Taraxacum officinale. J Ethnopharmacol. 2008;115(1): 82-88.

18. Qureshi S, Banday MT, Adil S, Shakeel I, Munshi ZH. Effect of dandelion leaves and fenugreek seeds with or without enzyme addition on performance and blood biochemistry of broiler chicken and evaluation of their in vitro antibacterial activity. Indian J Anim Sci. 2015;85(11):1248-1254.

19. Samolińska W, Grela ER, Kowalczuk-Vasilev E, Kiczorowska B, Klebaniuk R, Hanczakowska E. Evaluation of garlic and dandelion supplementation on the growth performance, carcass traits, and fatty acid composition of growing-finishing pigs. Anim Feed Sci Technol. 2020;259:114316.

20. Tan X, Sun Z. Dietary dandelion extract improved growth performance, immunity, intestinal morphology and microbiota composition of golden pompano Trachinotus ovatus. Aquacult Rep. 2020;18:100491.

21. Tan X, Sun Z, Zhou C, Zhong H, Wang A. Effects of dietary dandelion extract on intestinal morphology, antioxidant status, immune function and physical barrier function of juvenile golden pompano Trachinotus ovatus. Fish Shellfish Immunol. 2018;73:197-206.

22. Alhotan RA, Sulaiman A, Alharthi AS, Abudabos AM. Protective influence of betaine on intestinal health by regulating inflammation and improving barrier function in broilers under heat stress. Poult Sci.

2021;100(9):101337.

23. Shivatare RS, Phopase ML, Nagore DH, Nipanikar SU, Chitlange SS. Development and Validation of HPLC Analytical Protocol for Quantification of Salicin from Salix alba L. Inventi Impact: Pharm Anal Qual Assur. 2014.

24. Qureshi S, Adil S, Abd El-Hack ME, Alagawany M, Farag MR. Beneficial uses of dandelion herb (Taraxacum officinale) in poultry nutrition. World's Poult Sci J. 2017;73(3):591-602. 
25. Kaur AP, Bhardwaj S, Dhanjal DS, Nepovimova E, Sen F. Plant Prebiotics and Their Role in the Amelioration of Diseases. Biomolecules. 2021;11(3):440

26. Willems OW, Miller SP, Wood BJ. Aspects of selection for feed efficiency in meat producing poultry. World's Poult Sci J. 2013;69(1):77-88.

27. Noor AS, Kadhim AH, Ali MA. The effect of feeding different levels of dandelion leaf powder (Taraxacum officinale) in the diet on the productive and physiological performance of broiler chickens, strain Ross-308. IOP Conference Series: Earth and Environmental Science. 2021;722(1):012002.

28. Geravand M, Sharifi SD, Yaghobfar A, Mohammadi A, Hosseini SA, Ghazanfari S. Growth performance, ascites sensitivity, and ileal microbiota as affected by licorice essential oil in broiler chicken diets. Livest Sci. 2021;251:104670.

29. Venardou B, O'Doherty JV, Vigors S, O'Shea CJ, Sweeney T. Effects of dietary supplementation with a laminarinrich extract on the growth performance and gastrointestinal health in broilers. Poult Sci. 2021;100(7):101179.

30. Luissint AC, Parkos CA, Nusrat A. Inflammation and the intestinal barrier: leukocyte-epithelial cell interactions, cell junction remodeling, and mucosal repair. Gastroenterology. 2016;151(4):616-632.

31. Vicente Y, Da Rocha C, Yu J, Hernandez-Peredo G, Martinez L, Belen Pérez-Mies, et al. Architecture and function of the gastroesophageal barrier in the piglet. Dig Dis Sci. 2001,46(9):1899-1908.

32. Gonzalez-Mariscal L, Betanzos A, Nava P, Jaramillo BE. Tight junction proteins. Prog Biophys Mol Biol. 2003;81(1):1-44.

33. Long S, Piao X. Effects of dietary Forsythia suspensa extract supplementation to lactating sows and nursery pigs on post-weaning performance, antioxidant capacity, nutrient digestibility, immunoglobulins and intestinal health. J Anim Sci. 2021;99(8): skab142-skab142.

34. Lin JC, Wu JQ, Wang F, Tang FY, Sun J, Xu B, et al. QingBai decoction regulates intestinal permeability of dextran sulphate sodium-induced colitis through the modulation of notch and NF-KB signalling. Cell proliferation. 2019;52(2):e12547.

35. Piche T, Barbara G, Aubert P, Varannes S, Neunlist M. Impaired intestinal barrier integrity in the colon of patients with irritable bowel syndrome: involvement of soluble mediators. Gut. 2009;58(2):196-201.

36. Ahmad R, Sorrell MF, Batra SK, Dhawan P, Singh, AB. Gut permeability and mucosal inflammation: bad, good or context dependent. Mucosal Immunol. 2017;10(2):307-317.

37. McGuckin MA, Lindén SK, Sutton P, Florin TH. Mucin dynamics and enteric pathogens. Nat Rev Microbiol. 2011;9(4):265-278.

38. Mantis NJ, Rol N, Corthésy B. Secretory IgA's complex roles in immunity and mucosal homeostasis in the gut. Mucosal immunol. 2011;4(6): 603-611.

39. Pabst O. New concepts in the generation and functions of IgA. Nat Rev Immunol. 2012;12(12):821-832.

40. Liao L, Li J, Li J, Huang Y, Wu Y. Effects of Astragalus polysaccharides on intestinal morphology and intestinal immune cells of Muscovy ducklings infected with Muscovy duck reovirus. Poult Sci. 2021,100(1):64-72.

41. Song J, Li Q, Everaert N, Liu R, Zheng M, Zhao G, et al. Effects of inulin supplementation on intestinal barrier function and immunity in specific pathogen-free chickens with Salmonella infection. J Anim Sci. 2020;98(1):skz396.

42. Yegani M, Korver DR.. Factors affecting intestinal health in poultry. Poult Sci. 2008;87(10):2052-2063.

43. Valdes AM, Walter J, Segal E, Spector TD. Role of the gut microbiota in nutrition and health. Bmj. 2018;361. 
44. Flint HJ, Scott KP, Louis P, Duncan SH. The role of the gut microbiota in nutrition and health. Nat Rev Gastroenterol Hepatol. 2012;9(10):577-589.

45. Xue F, Shi L, Li Y, Ni A, Chen J. Effects of replacing dietary Aureomycin with a combination of plant essential oils on production performance and gastrointestinal health of broilers. Poult Sci. 2020;99(9):4521-4529.

46. Liu YS, Li S, Wang XF, Xing T, Gao F. Microbiota populations and short-chain fatty acids production in cecum of immunosuppressed broilers consuming diets containing Y-irradiated Astragalus polysaccharides. Poult Sci. 2021;100(1): 273-282.

47. Wang, Hong-Bin. Cellulase-assisted extraction and antibacterial activity of polysaccharides from the dandelion Taraxacum officinale. Carbohydr Polym. 2014;103:140-142.

48. Qian L, Zhou Y, Teng Z, Du CL, Tian C. Preparation and antibacterial activity of oligosaccharides derived from dandelion. Int J Biol Macromol. 2014;64:392-394.

49. Liu ZY, Wang XL, Ou SQ, Hou DX, He JH. Sanguinarine modulate gut microbiome and intestinal morphology to enhance growth performance in broilers. PloS one. 2020;15(6): e0234920.

50. Rooks MG, Garrett WS. Gut microbiota, metabolites and host immunity. Nat Rev Immunol. 2016;16(6):341352.

51. Morrison DJ, Preston T. Formation of short chain fatty acids by the gut microbiota and their impact on human metabolism. Gut microbes. 2016;7(3):189-200.

52. Neu J. Gastrointestinal maturation and implications for infant feeding. Early Hum Dev. 2007;83(12):767-775.

\section{Figures}




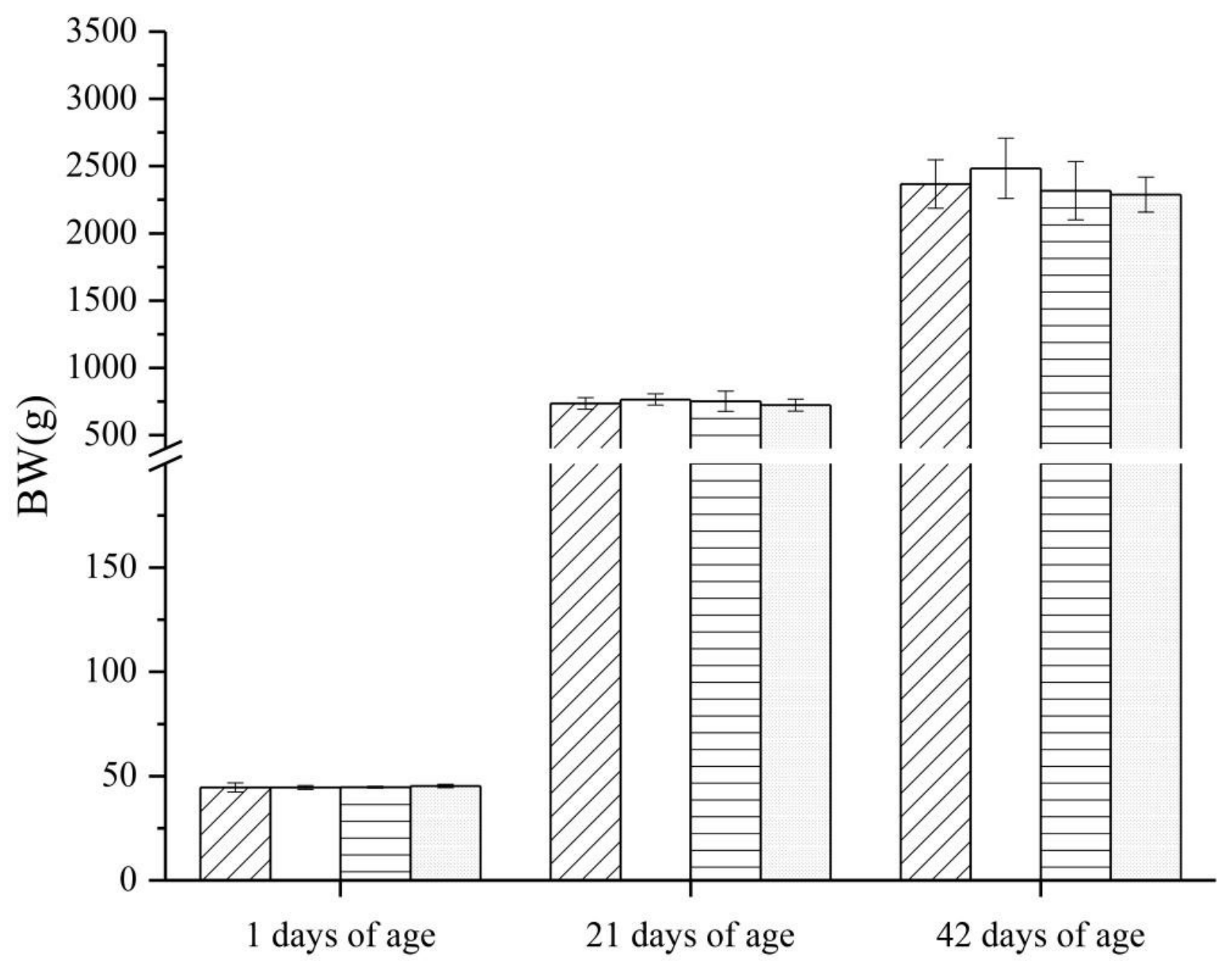

Figure 1

Effects of dandelion on body weight (BW) of broilers. CON = the control treatment; $L D=$ the low dose of dandelion treatment; $\mathrm{HD}=$ the high dose of dandelion treatment; $\mathrm{CTC}=$ the chlortetracycline treatment. The values are expressed as mean + SEM $(n=5)$. 


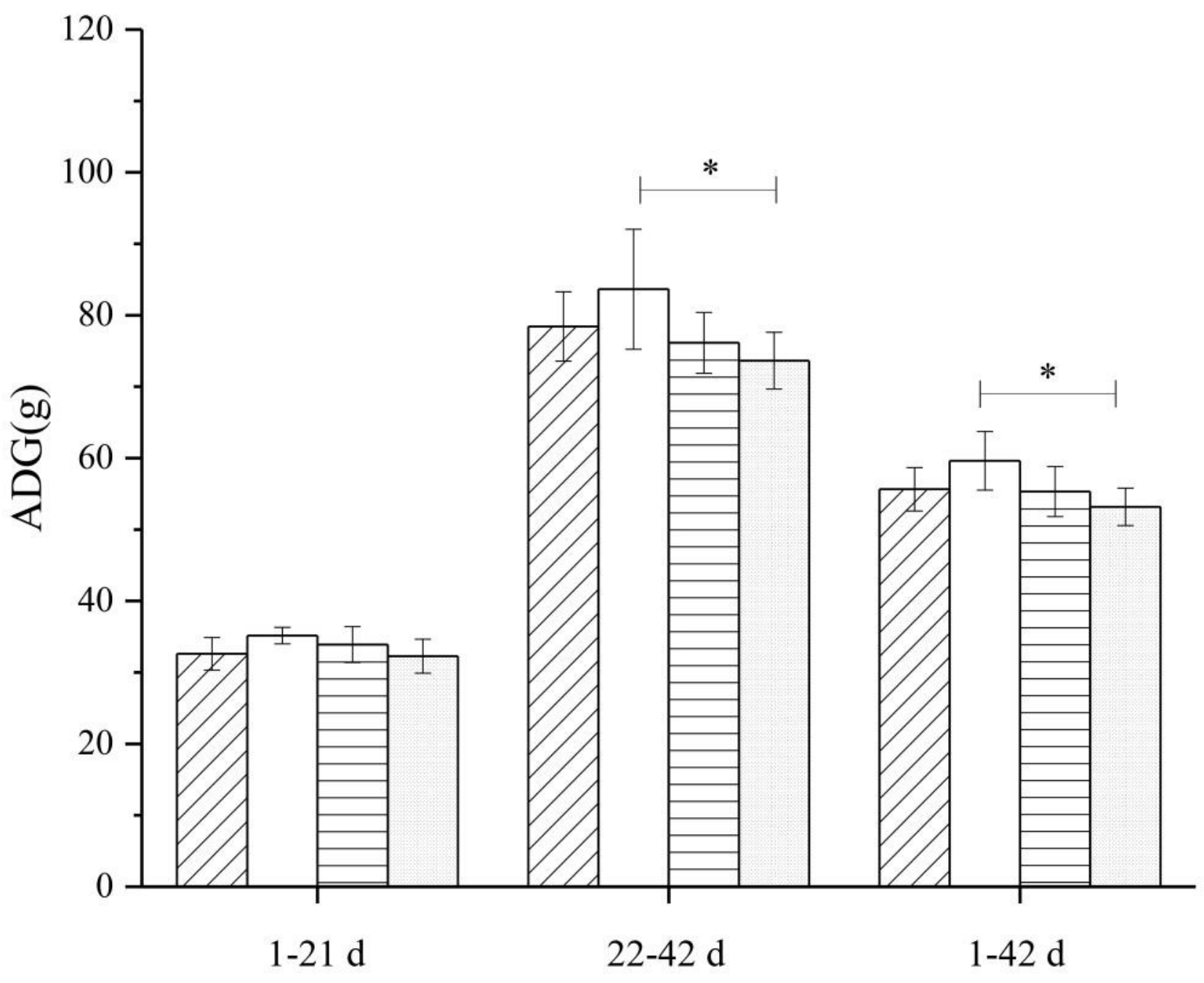

Figure 2

Effects of dandelion on average daily gain (ADG) of broilers. $C O N=$ the control treatment; $L D=$ the low dose of dandelion treatment; $\mathrm{HD}=$ the high dose of dandelion treatment; $\mathrm{CTC}=$ the chlortetracycline treatment. The values are expressed as mean + SEM $(n=5)$. ${ }^{*}$ Significantly different from CTC.

\section{Figure 3}

Effects of dandelion on average daily feed intake (ADFI) of broilers. $C O N=$ the control treatment; $L D=$ the low dose of dandelion treatment; $\mathrm{HD}=$ the high dose of dandelion treatment; $\mathrm{CTC}=$ the chlortetracycline treatment. The values are expressed as mean + SEM $(n=5) .{ }^{a-b}$ No significant difference between common superscript letters between CON, LD and HD $(P<0.05)$. *Significantly different from CTC. 


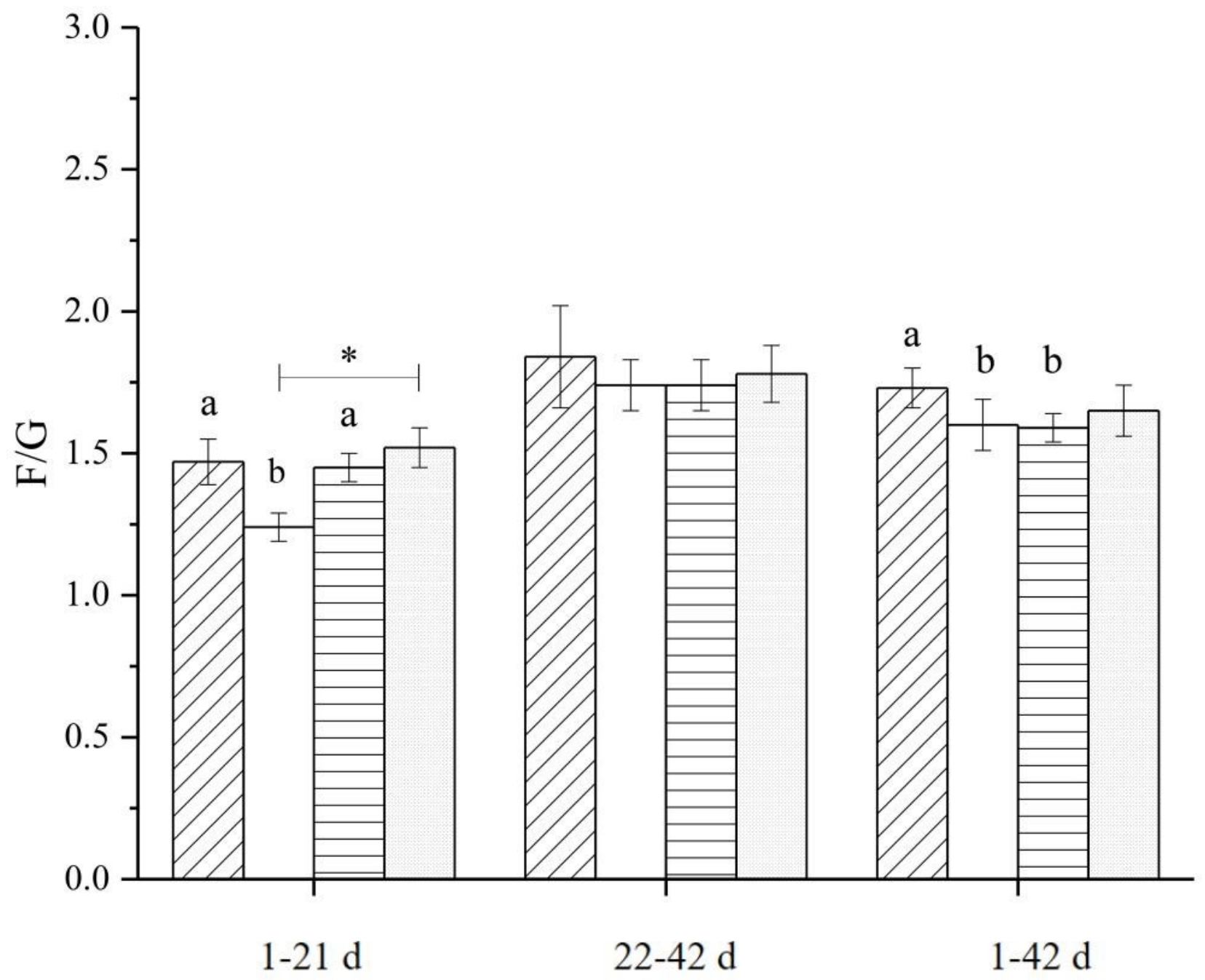

Figure 4

Effects of dandelion on feed to gain ratio $(F / G)$ of broilers. CON = the control treatment; $L D=$ the low dose of dandelion treatment; $\mathrm{HD}=$ the high dose of dandelion treatment; $\mathrm{CTC}=$ the chlortetracycline treatment. The values are expressed as mean + SEM $(n=5)$. ${ }^{a-b}$ No significant difference between common superscript letters between CON, LD and $\mathrm{HD}(P<0.05)$. *Significantly different from CTC. 


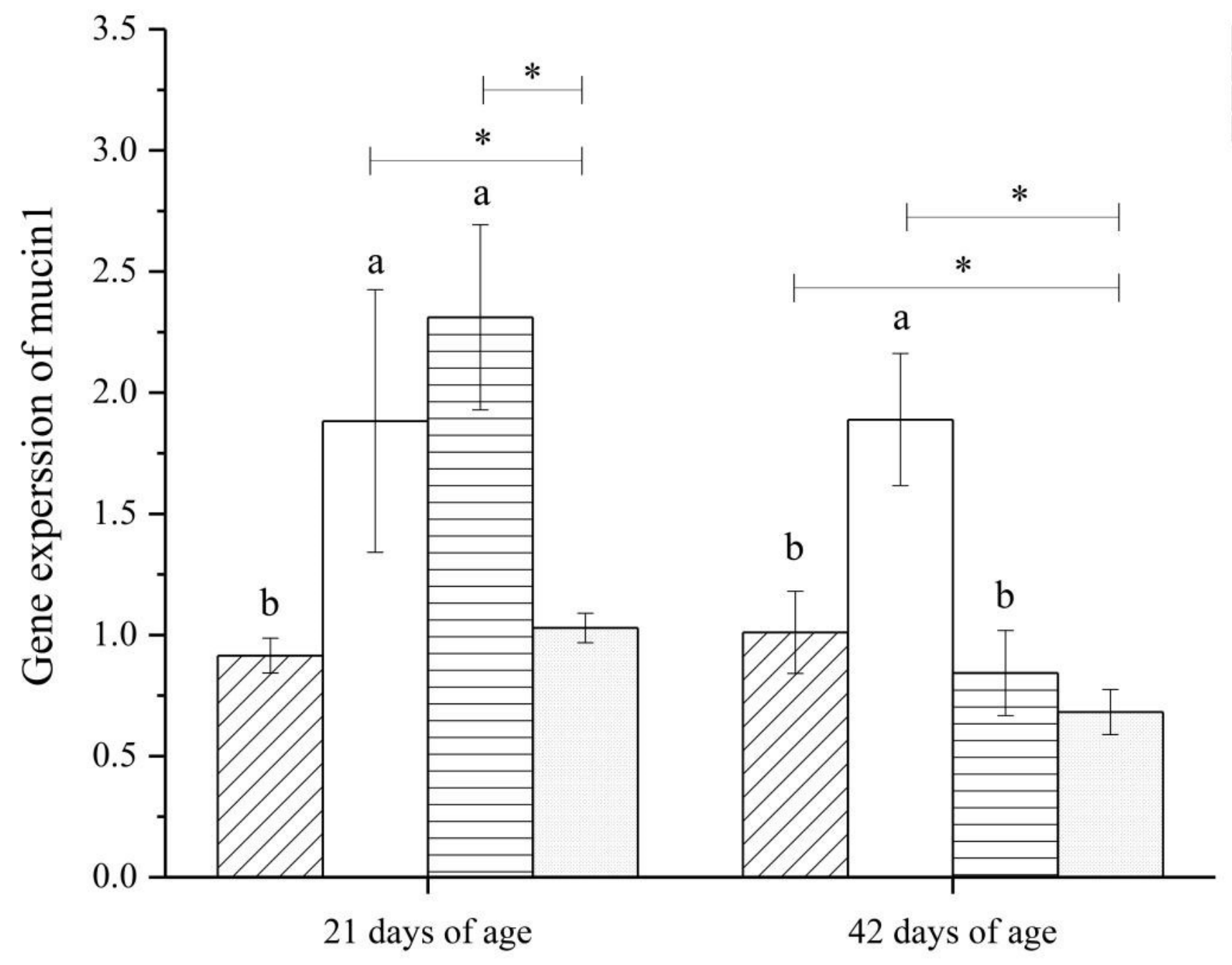

Figure 5

Effects of dandelion on gene expression of mucin1. $C O N=$ the control treatment; $L D=$ the low dose of dandelion treatment; $\mathrm{HD}=$ the high dose of dandelion treatment; CTC = the chlortetracycline treatment. The values are expressed as mean + SEM $(n=5) .{ }^{a-b}$ No significant difference between common superscript letters between CON, $\mathrm{LD}$ and $\mathrm{HD}(P<0.05)$. ${ }^{*}$ Significantly different from CTC. 
PCoA - PC1 vs PC2

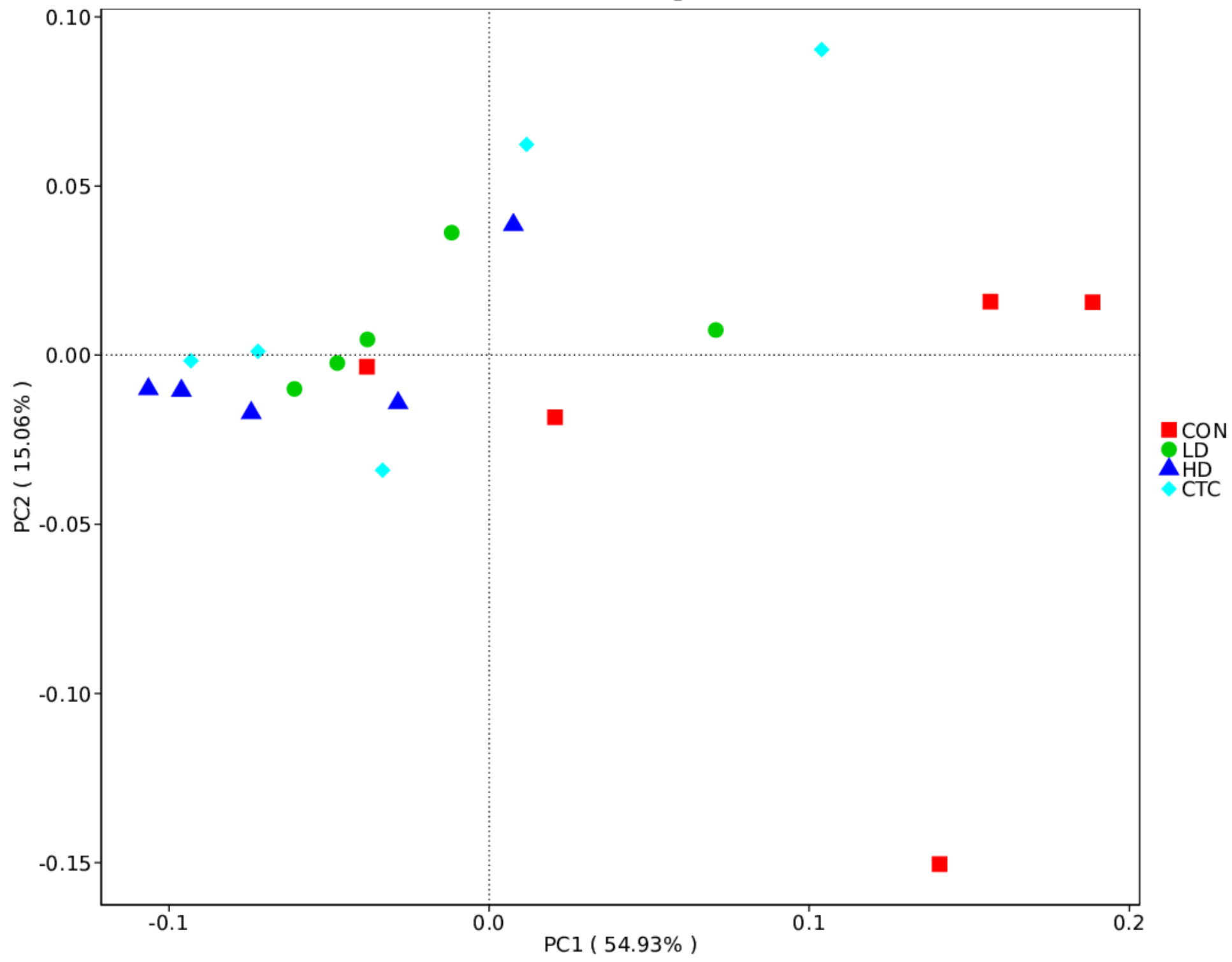

Figure 6

Principal coordinate analysis on community structures of the ileal microbiota in the different treatments (21 days old). $\mathrm{CON}=$ the control treatment; $\mathrm{LD}=$ the low dose of dandelion treatment; $\mathrm{HD}=$ the high dose of dandelion treatment; CTC $=$ the chlortetracycline treatment. 
PCoA - PC1 vs PC2

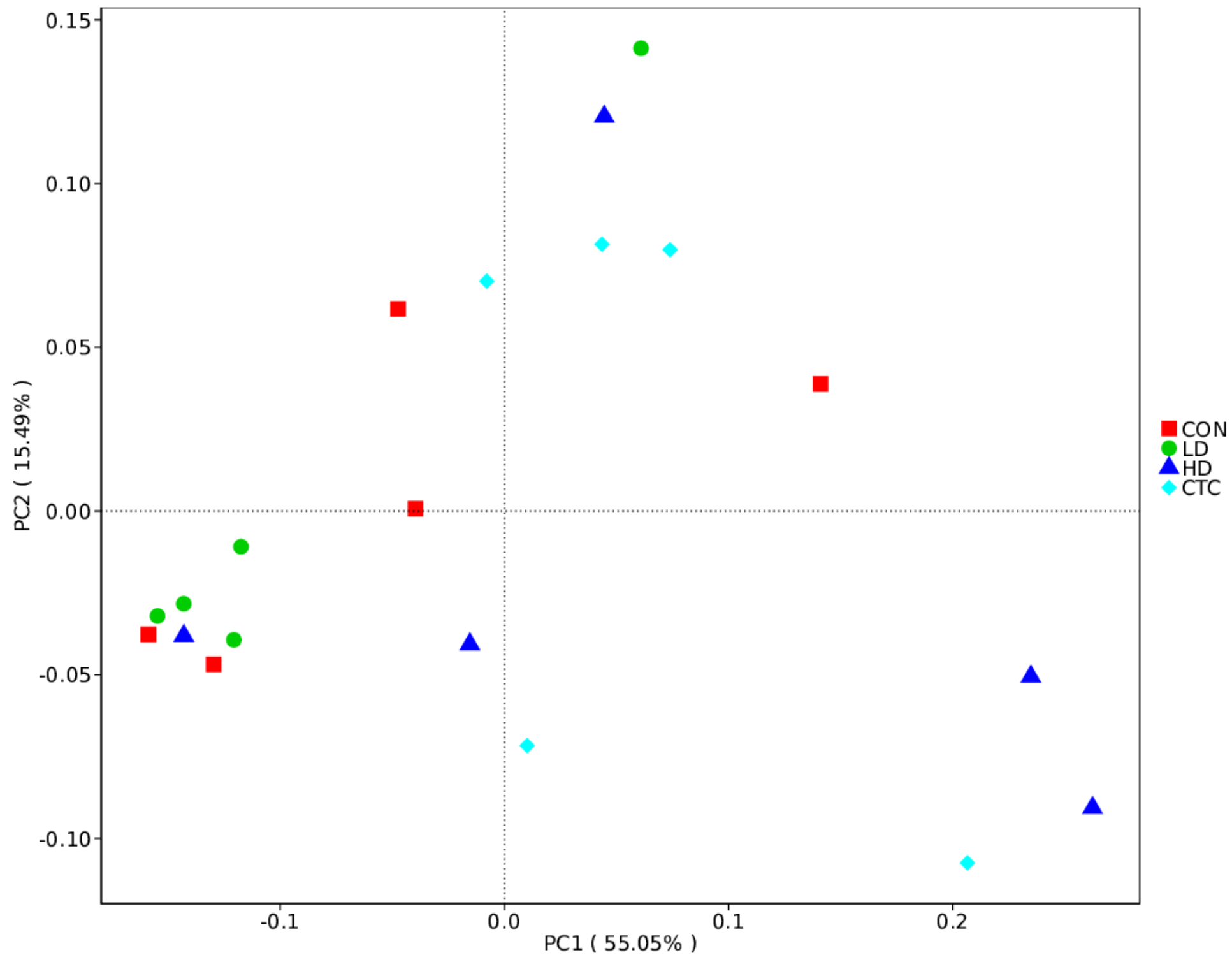

Figure 7

Principal coordinate analysis on community structures of the ileal microbiota in the different treatments (42 days old). $\mathrm{CON}=$ the control treatment; $\mathrm{LD}=$ the low dose of dandelion treatment; $\mathrm{HD}=$ the high dose of dandelion treatment; CTC = the chlortetracycline treatment. 